Proc. Indian Acad. Sci. (Math. Sci.) Vol. 113, No. 2, May 2003, pp. 139-152.

Printed in India

\title{
The Jacobian of a nonorientable Klein surface
}

\author{
PABLO ARÉS-GASTESI and INDRANIL BISWAS \\ School of Mathematics, Tata Institute of Fundamental Research, Mumbai 400 005, \\ India \\ E-mail: pablo@math.tifr.res.in; indranil@math.tifr.res.in
}

MS received 10 July 2002; revised 17 January 2003

\begin{abstract}
Using divisors, an analog of the Jacobian for a compact connected nonorientable Klein surface $Y$ is constructed. The Jacobian is identified with the dual of the space of all harmonic real one-forms on $Y$ quotiented by the torsion-free part of the first integral homology of $Y$. Denote by $X$ the double cover of $Y$ given by orientation. The Jacobian of $Y$ is identified with the space of all degree zero holomorphic line bundles $L$ over $X$ with the property that $L$ is isomorphic to $\sigma^{*} \bar{L}$, where $\sigma$ is the involution of $X$.
\end{abstract}

Keywords. Nonorientable surface; divisor; Jacobian

\section{Introduction}

Let $Y$ be a compact connected nonorientable Riemann surface, that is, each transition function is either holomorphic or anti-holomorphic. We consider surfaces without boundary. Let $X$ denote the double cover of $Y$ given by the local orientations. So $X$ is a compact connected Riemann surface.

In $\$ 2$. we define a morphism from $Y$ to $\overline{\mathbb{H}}$, the closure of the upper half-plane in the Riemann sphere $\widehat{\mathbb{C}}$. Let $\operatorname{Div}_{0}(Y)$ denote the group defined by all formal finite sums of the form $\sum n_{i} y_{i}$, where $n_{i} \in \mathbb{Z}$ with $\sum n_{i}=0$ and $y_{i} \in Y$. We call such a divisor $D$ to be principal if there is a morphism (see 2 . for the definition of morphism) $u$ from $Y$ to $\overline{\mathbb{H}}$ with the property that

$$
D=u^{-1}(0)-u^{-1}(\infty) .
$$

Let $J_{0}(Y)$ denote the quotient of $\operatorname{Div}_{0}(Y)$ by its subgroup consisting of all principal divisors. This $J_{0}(Y)$ is the analog of the Jacobian for a nonorientable Riemann surface.

Harmonic one-forms are defined on $Y$. Let $H_{\mathbb{R}}^{1}(Y)$ denote the space of all harmonic real one-forms on $Y$. The torsion-free part of $H_{1}(Y, \mathbb{Z})$ is a subgroup of $\mathscr{H}_{\mathbb{R}}^{1}(Y)^{*}$. The quotient is identified with $J_{0}(Y)$. This is proved by showing that $\mathscr{H}_{\mathbb{R}}^{1}(Y)$ is identified with the space of all holomorphic one-forms $\omega$ on $X$ satisfying the identity $\bar{\omega}=\sigma^{*} \omega$, where $\sigma$ is the nontrivial automorphism of the double cover $X$ of $Y$ (Theorem 2.7).

For a holomorphic line bundle $L$ over $X$, the pullback $\sigma^{*} \bar{L}$ is again a holomorphic line bundle over $X$. We show that $J_{0}(Y)$ is identified with the group of all holomorphic line bundles $L$ over $X$ for which the holomorphic line bundle $\sigma^{*} \bar{L}$ is isomorphic to $L$ (Theorem 4.2).

A compact Riemann surface is a smooth projective curve over $\mathbb{C}$. Conversly, every smooth projective curve over $\mathbb{C}$ corresponds to a compact Riemann surface. If we take a 
smooth projective curve $X_{\mathbb{R}}$ defined over $\mathbb{R}$, then using the inclusion of $\mathbb{R}$ in $\mathbb{C}$ we get a smooth projective curve $X_{\mathbb{C}}$ over $\mathbb{C}$. Now, since the involution of $\mathbb{C}$ defined by conjugation fixes $\mathbb{R}$, the complex curve $X_{\mathbb{C}}$ is equipped with an anti-holomorphic involution that reverses the orientation. Conversely, every complex projective curve equipped with an anti-holomorphic involution is actually defined over $\mathbb{R}$. If the involution does not have any fixed points, that is, the curve does not have any real points, then it is called an imaginary curve.

Therefore, a nonorientable Riemann surface $Y$ (without boundary) corresponds to an imaginary algebraic curve defined over $\mathbb{R}$. The Jacobian of the complexification $Y \mathbb{C}$ is also the complexification of a variety defined over $\mathbb{R}$. The Jacobian $J_{0}(Y)$ coincides with this variety defined over $\mathbb{R}$.

\section{Divisors on a nonorientable surface}

Let $Y$ be a compact connected nonorientable surface. In other words, $Y$ is a compact connected nonorientable smooth manifold of dimension two, and $Y$ has a covering by smooth coordinate charts such that each transition function is either holomorphic or antiholomorphic. Any coordinate chart in the maximal atlas satisfying the above condition on transition functions will be called compatible. Such a nonorientable surface is called a Klein surface.

\section{DEFINITION 2.1.}

A divisor $D$ on $Y$ is a formal sum of type

$$
D=\sum_{y \in Y} n_{y} y,
$$

where $n_{y} \in \mathbb{Z}$ and $n_{y}=0$ except for a finitely many points of $Y$.

\section{DEFINITION 2.2.}

The degree of a divisor $D=\sum_{y \in Y} n_{y} y$ is defined to be the integer $\operatorname{deg}(D):=\sum_{y \in Y} n_{y}$.

We will denote by $\operatorname{Div}(Y)$ the set of all divisors on $Y$. Let $\operatorname{Div}_{d}(Y) \subset \operatorname{Div}(Y)$ be the divisors of degree $d$.

Let $\pi: X \rightarrow Y$ be a double cover of $Y$ given by local orientations on $Y$. So for a contractible open subset $U \subset Y$, the inverse image $\pi^{-1}(U)$ is two copies of $U$ with the two possible orientations on $U$ (see [1] for more details on Klein surfaces and their double covers).

Therefore, $X$ is a Riemann surface, and the change of orientation defines an antiholomorphic involution $\sigma: X \rightarrow X$ that commutes with $\pi$.

The involution $\sigma$ induces in a natural way a mapping on the set of divisors on the Riemann surface $X$ as follows

$$
\begin{aligned}
\sigma^{*}: \operatorname{Div}(X) & \longrightarrow \operatorname{Div}(X) \\
\sum m_{j} x_{j} & \longmapsto \sum m_{j} \sigma\left(x_{j}\right) .
\end{aligned}
$$

Observe that $\sigma^{*}$ preserves the degree.

Similarly, the quotient map $\pi: X \rightarrow Y$ induces mappings between the divisors on $X$ and $Y$. To define those mappings we first set up some notation. For any point $y \in Y$ we will 
denote by $\pi^{-1}(y)$ the divisor given by the inverse image of $y$. In other words, $\pi^{-1}(y)=$ $x+\sigma(x)$, where $x \in X$ is a point satisfying $\pi(x)=y$. Then we can define two mappings as follows:

$$
\begin{aligned}
\pi^{*}: \operatorname{Div}(Y) & \rightarrow \operatorname{Div}(X), \quad \pi_{*}: \operatorname{Div}(X) \rightarrow \operatorname{Div}(Y), \\
\sum_{j=1}^{s} n_{j} y_{j} & \mapsto \sum_{j=1}^{s} n_{j} \pi^{-1}\left(y_{j}\right), \quad \sum_{j=1}^{s} m_{j} x_{j} \mapsto \sum_{j=1}^{s} m_{j} \pi\left(x_{j}\right) .
\end{aligned}
$$

Observe that $\left(\pi_{*} \circ \pi^{*}\right)(D)=2 D$ and $\left(\pi^{*} \circ \pi_{*}\right)(E)=E+\sigma^{*}(E)$ for $D \in \operatorname{Div}(Y)$ and $E \in$ $\operatorname{Div}(X)$.

Let $\operatorname{Div}(X)^{\sigma^{*}}$ denote the set of fixed points of $\sigma^{*}$ on $\operatorname{Div}(X)$.

The following lemma follows immediately from the above definitions.

Lemma 2.3. The group $\operatorname{Div}(Y)$ is identified with $\operatorname{Div}(X)^{\sigma^{*}}$. The isomorphism takes the subgroup $\operatorname{Div}_{0}(Y)$ to $\operatorname{Div}(X)_{0}^{\sigma^{*}}=\operatorname{Div}_{0}(X) \cap \operatorname{Div}(X)^{\sigma^{*}}$.

Let $j: \widehat{\mathbb{C}} \rightarrow \widehat{\mathbb{C}}$ denote the mapping induced by conjugation on the Riemann sphere $\widehat{\mathbb{C}}$, so that $j(z)=\bar{z}$ and $j(\infty)=\infty$. The quotient space is a surface with boundary, $\overline{\mathbb{H}}=\widehat{\mathbb{C}} /\langle j\rangle$. We can also identify $\overline{\mathbb{H}}$ with the closure of $\mathbb{H}$ (the upper half-plane) in the Riemann sphere. Let

$$
p: \widehat{\mathbb{C}} \longrightarrow \overline{\mathbb{H}}
$$

denote the quotient map. After identifying $\overline{\mathbb{H}}$ with the closure of $\mathbb{H}$ the map $p$ coincides with the one defined by $p(x+\sqrt{-1} y)=x+\sqrt{-1}|y|$ and $p(\infty)=\infty$.

A morphism from $Y$ to $\overline{\mathbb{H}}$ is a continuous mapping

$$
u: Y \longrightarrow \overline{\mathbb{H}}
$$

such that if $(U, w)$ is a local coordinate function defined on $Y$, compatible with the Riemann surface structure, with $w(U) \subset \mathbb{H}$, then there exists a holomorphic function $F$ : $w(U) \rightarrow \mathbb{C}$ that makes the following diagram commutative:

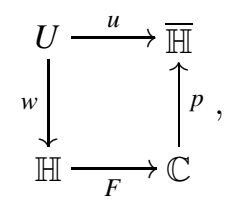

where $p$ is defined above.

Let $u$ be a morphism, as above, from $Y$ to $\overline{\mathbb{H}}$ which is not identically equal to 0 or $\infty$. If $z_{0}$ is a point of $\overline{\mathbb{H}}$, then by $u^{-1}\left(z_{0}\right)$ we understand the divisor given by the inverse image of $z_{0}$ under $u$ (so the integers $n_{j}$ in Definition 2.1 are given by the multiplicities of $u$ at the corresponding points). Since 0 and $\infty$ in $\widehat{\mathbb{C}}$ project to two different points on $\overline{\mathbb{H}}$,

$$
\operatorname{div}(u):=u^{-1}(0)-u^{-1}(\infty) \in \operatorname{Div}(Y)
$$

is a divisor on $Y$.

\section{DEFINITION 2.4.}

A divisor $D \in \operatorname{Div}(Y)$ is called principal if $D=\operatorname{div}(u)$ for some morphism $u: Y \rightarrow \overline{\mathbb{H}}$ of the above type. The set of principal divisors of $Y$ will be denoted by $\operatorname{Div}_{P}(Y)$. 


\section{PROPOSITION 2.5.}

A divisor $D$ on $Y$ is principal if and only if there exists a divisor $E \in \operatorname{Div}_{P}(X) \cap \operatorname{Div}(X)^{\sigma^{*}}$ with $\pi^{*} D=E$.

Proof. Let $E=\operatorname{div}(f)$ be a principal divisor in $\operatorname{Div}(X)^{\sigma^{*}}$, where $f$ is a non-constant meromorphic function on $X$. Consider the function $\psi$ on $X$ defined by $\psi(x)=\overline{f(\sigma(x))}$ on $X$. This function $\psi$ is clearly meromorphic.

Since $E \in \operatorname{Div}(X)^{\sigma^{*}}$, we have $\operatorname{div}(\psi)=\operatorname{div}(f)$. Consequently, there exists a constant $c \in \mathbb{C} \backslash\{0\}$ such that $\psi=c f$.

Therefore, we have $f(x)=\psi(x) / c=\overline{f(\sigma(x))} / c=\overline{\psi(\sigma(x))} /|c|^{2}=f(x) /|c|^{2}$. Take $c_{0} \in \mathbb{C}$ with $c_{0}^{2}=c$. Set $f_{0}=c_{0} f$.

The divisor for the meromorphic function $f_{0}$ coincides with $E$. Furthermore, $f_{0}$ satisfies the condition

$$
f_{0} \circ \sigma=\overline{f_{0}} .
$$

Therefore, it induces a map

$$
\hat{f}: Y:=X / \sigma \longrightarrow \overline{\mathbb{H}}:=\widehat{\mathbb{C}} /\langle j\rangle
$$

with $\operatorname{div}(\hat{f})=D$.

Conversely, let $D=\operatorname{div}(u)$ be a principal divisor on $Y$. Consider the composition $u \circ \pi$ : $X \longrightarrow \overline{\mathbb{H}}$. It is straight-forward to see that the function $u \circ p$ lifts to a smooth function

$$
f: X \longrightarrow \widehat{\mathbb{C}}
$$

such that $p \circ f=u \circ \pi$. There are two such smooth lifts; one is holomorphic and the other is anti-holomorphic ( $u \circ p$ also has a continuous lift, defined by the inclusion of $\overline{\mathbb{H}}$ in $\widehat{\mathbb{C}}$ which is not smooth). Let $f$ denote the holomorphic one. Since $\operatorname{div}(f)=\pi^{*}(D) \in$ $\operatorname{Div}(X)^{\sigma^{*}}$, the proof of the proposition is complete.

\section{DEFINITION 2.6.}

The quotient of $\operatorname{Div}_{0}(Y)$, the group of all degree zero divisors on $Y$, by the subgroup of all principal divisors on $Y$ is called the Jacobian of $Y$. The Jacobian of $Y$ will be denoted by $J_{0}(Y)$.

From Proposition 2.5 it follows immediately that by sending any divisor $D$ on $Y$ to the divisor $\pi^{*} D$ on $X$ we obtain an injective homomorphism from $J_{0}(Y)$ to the Jacobian $J_{0}(X)$ of $X$. From Lemma 2.3 it follows that $J_{0}(Y)$ coincides with the fixed point set of the involution of $J_{0}(X)$ defined by $\sigma$.

A function $f: W \rightarrow \mathbb{R}$, defined on an open subset of $Y$ is called harmonic if for every point $y \in W$, there exists a compatible coordinate chart $(U, w)$, with

$$
y \in U \subseteq W,
$$

such that the function $f \circ w^{-1}$ is harmonic. Since precomposition with holomorphic and anti-holomorphic functions preserve harmonicity, we conclude that harmonic functions are well-defined on $Y$.

We say that a real one-form $\eta$ on $Y$ is harmonic if it is locally given by $\mathrm{d} f$, where $f$ is a harmonic function. 
Let $\Omega$ denote the holomorphic cotangent bundle of the Riemann surface $X$. If $\omega \in$ $H^{0}(X, \Omega)$ is given locally by $\omega=f \mathrm{~d} z$, where $f$ is a holomorphic function, then define

$$
\overline{\sigma^{*} \omega}:=(\overline{f \circ \sigma}) \mathrm{d}(\bar{z} \circ \sigma) .
$$

So if $\omega$ is defined over $U$, then $\overline{\sigma^{*} \omega}$ is a holomorphic one-form defined over $\sigma(U)$. More generally, for a one-form $\alpha=u \mathrm{~d} z+v \mathrm{~d} \bar{z}$, set

$$
\sigma^{*} \alpha=(u \circ \sigma) \mathrm{d}(z \circ \sigma)+(\nu \circ \sigma) \mathrm{d}(\bar{z} \circ \sigma) .
$$

Let $\mathscr{H}_{\mathbb{R}}^{1}(Y)$ and $\mathscr{H}_{\mathbb{R}}^{1}(X)$ denote the space of all real harmonic one-forms on $Y$ and $X$ respectively. Using the map $\pi: X \rightarrow Y$, we can lift harmonic forms on $Y$ to smooth forms on $X$. It is easy to see that the pullback of a harmonic form on $Y$ is a harmonic form on $X$. Therefore, there is a well-defined injective homomorphism $\pi^{*}: \mathscr{H}_{\mathbb{R}}^{1}(Y) \longrightarrow \mathscr{H}_{\mathbb{R}}^{1}(X)$.

The complex structure on $X$ defines a Hodge-* operator on one-forms on $X$. In local holomorphic coordinates the Hodge- $*$ operator is

$$
*(u \mathrm{~d} z+v \mathrm{~d} \bar{z})=-\sqrt{-1} u \mathrm{~d} z+\sqrt{-1} v \mathrm{~d} \bar{z}
$$

or $*(a \mathrm{~d} x+b \mathrm{~d} y)=-b \mathrm{~d} x+a \mathrm{~d} y$.

A holomorphic one-form $\omega$ on $X$ will be called $\sigma$-invariant if $\sigma^{*} \omega=\bar{\omega}$. The space of all $\sigma$-invariant forms on $X$ will be denoted by $H^{0}(X, \Omega)^{\overline{\sigma^{*}}}$.

Theorem 2.7. A holomorphic form $\omega \in H^{0}(X, \Omega)$ is $\sigma$-invariant if and only if there exists a form $\eta \in \mathscr{H}_{\mathbb{R}}^{1}(Y)$ such that $\omega=\beta+\sqrt{-1}(* \beta)$, where $\beta=\pi^{*} \eta$.

The homomorphism $H_{\mathbb{R}}^{1}(Y) \longrightarrow H^{0}(X, \Omega)^{\overline{\sigma^{*}}}$ defined by

$$
\eta \longmapsto \pi^{*} \eta+\sqrt{-1}\left(* \pi^{*} \eta\right)
$$

is an isomorphism of real vector spaces.

Proof. Take any $\omega \in H^{0}(X, \Omega)$. Let $\omega=\beta+\sqrt{-1}(* \beta)$, where $\beta$ is a real one-form. Now the condition $\sigma^{*} \omega=\bar{\omega}$ immediately implies that $\sigma^{*} \beta=\beta$. Therefore, $\beta$ is the pullback of a form on $Y$. For any $\eta \in \mathscr{H}_{\mathbb{R}}^{1}(Y)$, the form $\pi^{*} \eta+\sqrt{-1}\left(* \pi^{*} \eta\right)$ is a $\sigma$ invariant holomorphic one-form.

Let

$$
\varphi: \mathscr{H}_{\mathbb{R}}^{1}(Y) \longrightarrow H^{0}(X, \Omega)^{\overline{\sigma^{*}}}
$$

be the homomorphism that sends any harmonic form $\eta \in \mathscr{H}_{\mathbb{R}}^{1}(Y)$ to the holomorphic form $\pi^{*} \eta+\sqrt{-1}\left(* \pi^{*} \eta\right)$. This homomorphism is injective since a holomorphic one-form with vanishing real part must be identically zero.

The inverse homomorphism

$$
H^{0}(X, \Omega)^{\overline{\sigma^{*}}} \longrightarrow \mathscr{H}_{\mathbb{R}}^{1}(Y)
$$

sends a $\sigma$-invariant form $\omega$ on $Y$ to $\eta$ with the property

$$
\pi^{*} \eta=\frac{\omega+\bar{\omega}}{2}
$$

This completes the proof of the theorem. 


\section{The Jacobian}

A closed oriented smooth path $\gamma$ on $X$ gives an element $L_{\gamma} \in H^{0}(X, \Omega)^{*}$ defined by

$$
L_{\gamma}(\omega)=\int_{\gamma} \omega,
$$

where $\omega \in H^{0}(X, \Omega)$. Using Stokes' theorem we get a mapping from $H_{1}(X, \mathbb{Z})$ to $H^{0}(X, \Omega)^{*}$. The quotient space $H^{0}(X, \Omega)^{*} / H_{1}(X, \mathbb{Z})$ will be denoted by $J_{1}(X)$.

As we saw in the previous section, for a holomorphic one-form $\omega$ on $X$, the form $\overline{\sigma^{*} \omega}$ is again a holomorphic one-form. This involution of $H^{0}(X, \Omega)$ induces an involution

$$
\sigma_{1}: H^{0}(X, \Omega)^{*} \longrightarrow H^{0}(X, \Omega)^{*}
$$

In other words, $\left(\sigma_{1}(L)\right)(\omega)=\overline{L\left(\overline{\sigma^{*}(\omega)}\right)}$. It is easy to check that for any closed smoothoriented path $\gamma$ on $X$, the identity

$$
\sigma_{1}\left(L_{\gamma}\right)=L_{\sigma(\gamma)}
$$

is valid. So, the involution $\sigma_{1}$ preserves the subgroup $H_{1}(X, \mathbb{Z}) \subset H^{0}(X, \Omega)^{*}$.

Consequently, the involution $\sigma_{1}$ of $H^{0}(X, \Omega)^{*}$ induces an involution on the quotient space $J_{1}(X)$. The involution of $J_{1}(X)$ obtained this way will also be denoted by $\sigma_{1}$.

Let $g$ be the genus of the compact connected Riemann surface $X$. Suppose we have a canonical basis of $H_{1}(X, \mathbb{Z})$, say $\left\{\alpha_{1}, \ldots, \alpha_{g}, \beta_{1}, \ldots, \beta_{g}\right\}$. This means that the corresponding intersection matrix is

$$
J=\left(\begin{array}{cc}
0 & -I \\
I & 0
\end{array}\right),
$$

where $I$ is the identity matrix of rank $g$. Then there exists a unique basis of $H^{0}(X, \Omega)$, say $\left\{\omega_{1}, \ldots, \omega_{g}\right\}$, such that $\int_{\alpha_{k}} \omega_{j}=\delta_{j k}([2]$, Proposition III.2.8). We say that this basis is adapted to the given basis of homology.

Using this adapted basis we can identify $H^{0}(X, \Omega)^{*}$ with $\mathbb{C}^{g}$ by sending the element $L$ of $H^{0}(X, \Omega)^{*}$ to the vector $\left(L\left(\omega_{1}\right), \ldots, L\left(\omega_{g}\right)\right)$.

Therefore, for any $\gamma \in H_{1}(X, \mathbb{Z})$, we may identify the element $L_{\gamma} \in H^{0}(X, \Omega)^{*}$ with

$$
\left(L_{\gamma}\left(\omega_{1}\right), \ldots, L_{\gamma}\left(\omega_{g}\right)\right) \in \mathbb{C}^{g} .
$$

Denote by $\mathscr{L}$ the lattice in $\mathbb{C}^{g}$ defined by $H_{1}(X, \mathbb{Z})$ using this identification. The quotient space $J_{1}(X)$ defined earlier is clearly identified with the quotient $\mathbb{C}^{g} / \mathscr{L}$.

Assume that the basis $\left\{\omega_{j}\right\}$ is $\sigma$-invariant, that is, $\overline{\sigma^{*}\left(\omega_{j}\right)}=\omega_{j}$ for each $j \in[1, g]$. It is easy to check that by the above isomorphism of $H^{0}(X, \Omega)^{*}$ with $\mathbb{C}^{g}$ the involution $\sigma_{1}$ of $H^{0}(X, \Omega)^{*}$ (defined earlier) coincides with the conjugation defined as $\left(z_{1}, \ldots, z_{g}\right) \longmapsto$ $\left(\overline{z_{1}}, \ldots, \overline{z_{g}}\right)$.

We will denote by $\sigma_{\#}$ the involution of $H_{1}(X, \mathbb{Z})$ induced by the involution $\sigma$ of $X$. Let

$$
\left\{\gamma_{1}, \ldots, \gamma_{g}, \delta_{1}, \ldots, \delta_{g}\right\}
$$

be a canonical basis of $H_{1}(X, \mathbb{Z})$ satisfying the condition $\sigma_{\#}\left(\gamma_{j}\right)=\gamma_{j}$ for all $j \in[1, g]$. Let $\left\{\omega_{1}, \ldots, \omega_{g}\right\}$ denote the corresponding adapted basis. 


\section{PROPOSITION 3.1.}

The above adapted basis $\left\{\omega_{1}, \ldots, \omega_{g}\right\}$ is $\sigma$-invariant.

Proof. Since

$$
\int_{\sigma_{\sharp} \gamma} \omega=\int_{\gamma} \sigma^{*} \omega=\overline{\int_{\gamma} \sigma^{*} \bar{\omega}}
$$

(as $\sigma$ is an involution), the proposition follows immediately.

As in $\$ 2$. let $J_{0}(X)$ denote the quotient $\operatorname{Div}_{0}(X) / \operatorname{Div}_{P}(X)$. For a meromorphic function $f$ we have $\sigma^{*}(\operatorname{div}(f))=\operatorname{div}(\overline{f \circ \sigma})$. So $\sigma^{*}$ induces an involution on $J_{0}(X)$. This involution of $J_{0}(X)$ will be denoted by $\sigma_{0}$.

Let $\left\{\omega_{1} \ldots, \omega_{g}\right\}$ be the basis in Proposition 3.1 Recall the quotient $J_{1}(X)$ of $H^{0}(X, \Omega)^{*}$ defined earlier. The Abel-Jacobi map $A: X \rightarrow J_{1}(X)$ is defined as follows: choose a point $x_{0}$ of $X$ and set $A(x)=\left[\int_{x_{0}}^{x} \omega_{1}, \ldots, \int_{x_{0}}^{x} \omega_{g}\right]$, where the brackets denote the equivalence class in $J_{1}(X)$. We have

$$
\begin{aligned}
A(\sigma(x))= & {\left[\int_{x_{0}}^{\sigma(x)} \omega_{1}, \ldots, \int_{x_{0}}^{\sigma(x)} \omega_{g}\right]=\left[\int_{x_{0}}^{\sigma\left(x_{0}\right)} \omega_{1}, \ldots, \int_{x_{0}}^{\sigma\left(x_{0}\right)} \omega_{g}\right] } \\
& +\left[\int_{\sigma\left(x_{0}\right)}^{\sigma(x)} \omega_{1}, \ldots, \int_{\sigma\left(x_{0}\right)}^{\sigma(x)} \omega_{g}\right] \\
= & c_{0}+\left[\int_{\sigma\left(x_{0}\right)}^{\sigma(x)} \sigma^{*}\left(\omega_{1}\right), \ldots, \int_{\sigma\left(x_{0}\right)}^{\sigma(x)} \sigma^{*}\left(\omega_{1}\right)\right] \\
= & c_{0}+\left[\int_{x_{0}}^{x} \overline{\omega_{1}}, \ldots, \int_{x_{0}}^{x} \overline{\omega_{g}}\right]=c_{0}+\overline{A(x)},
\end{aligned}
$$

where $c_{0}=A\left(\sigma\left(x_{0}\right)\right)$. For a divisor $D=\sum_{j=1}^{r} n_{j} x_{j}$, we define

$$
A(D)=\sum_{j=1}^{r} n_{j} A\left(x_{j}\right) .
$$

If $D$ has degree equal to 0 then we can write it as $D=\sum_{j=1}^{s} x_{j}-\sum_{j=1}^{s} y_{j}$, where $x_{j} \neq y_{k}$ (though we can have repetitions among the $x_{j} \mathrm{~s}$ or the $y_{k} \mathrm{~s}$ ). Then it is easy to check that

$$
A\left(\sigma_{0}(D)\right)=\overline{A(D)}=\sigma_{1}(A(D))
$$

where $\sigma_{1}$ and $\sigma_{0}$ are the earlier defined involutions of $H^{0}(X, \Omega)^{*}$ and $J_{0}(X)$ respectively.

By Abel's theorem, the map $A$ can be extended to a map from $J_{0}(X)$ to $J_{1}(X)$. By the Abel-Jacobi inversion problem, the map $A: J_{0}(X) \rightarrow J_{1}(X)$ is surjective. Thus (1) says that $\sigma_{0}$ and $\sigma_{1}$ are equivalent under $A$, that is, the following diagram commutes:

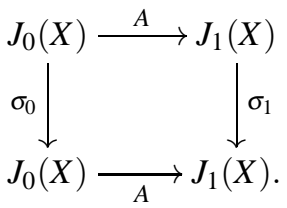


In the paragraph following Definition 2.6 we noted that the Jacobian $J_{0}(Y)$ coincides with the fixed point set of $J_{0}(X)$ for the action of the involution $\sigma_{0}$. Let $J_{1}(X)^{\sigma_{1}} \subset J_{1}(X)$ be the fixed point set for the action of the involution $\sigma_{1}$ on $J_{1}(X)$. From the commutativity of the diagram in (2) it follows immediately that $J_{0}(Y)$ is identified with $J_{1}(X)^{\sigma_{1}}$. Finally using Theorem 2.7 the Jacobian $J_{0}(Y)$ is identified with the quotient of $\mathscr{H}_{\mathbb{R}}^{1}(Y)$ by the torsion-free part of $H_{1}(Y, \mathbb{Z})$.

\section{Line bundles on a Klein surface}

Let $L$ be a holomorphic line bundle over a Riemann surface $X$. By $\bar{L}$ we will mean the $C^{\infty}$ complex line bundle over $X$ whose transition functions are the conjugations of the transition functions for $L$. To explain this, let $U_{i}, i \in I$, be an open covering of $X$ and assume that over each $U_{i}$ we are given a holomorphic trivialization of $L$. So for any ordered pair $i, j \in I$, we have the corresponding transition function

$$
f_{i, j}: U_{i} \cap U_{j} \longrightarrow \mathbb{C}^{*}
$$

which is holomorphic. The $C^{\infty}$ complex line bundle $\bar{L}$ has $C^{\infty}$ trivializations over each $U_{i}$, $i \in I$, and for any ordered pair $i, j \in I$ the corresponding transition function is $\overline{f_{i, j}}$. It is easy to see that the collection $\left\{\overline{f_{i, j}}\right\}_{i, j \in I}$ satisfy the cocycle condition to define a $C^{\infty}$ complex line bundle.

The line bundle $\bar{L}$ can also be defined without using local trivializations. A $C^{\infty}$ complex line bundle is a $C^{\infty}$ real vector bundle of rank two together with a smoothly varying complex structure on the fibers (which are real vector spaces of dimension two). The underlying real vector bundle of rank two for $\bar{L}$ coincides with the one for $L$. For any $x \in X$, if $J_{x}$ is the complex structure on the fiber $L_{x}$, then the complex structure of the fiber $\bar{L}_{x}$ is $-J_{x}$.

As in $\$ 2$. let $Y$ be a nonorientable Klein surface and $X$ its double cover, which is a connected Riemann surface of genus $g$.

Let $L$ be a holomorphic line bundle over $X$. The complex line bundle $\sigma^{*} \bar{L}$ has a natural holomorphic structure, where $\sigma$, as before, is the involution of $X$. To construct the holomorphic structure on $\sigma^{*} \bar{L}$, observe that if $f$ is a holomorphic function on an open subset $U$ of $X$, then $\overline{f \circ \sigma}$ is a holomorphic function of $\sigma(U)$. We can choose the above open subsets $U_{i}$ (sets over which $L$ is trivialized) in such a way that $\sigma\left(U_{i}\right)=U_{i}$. Now, since each $\overline{f_{i, j} \circ \sigma}$ is a holomorphic function on $U_{i} \cap U_{j}$, the complex line bundle $\sigma^{*} \bar{L}$ gets equipped with a holomorphic structure.

\section{PROPOSITION 4.1.}

Let $D$ be a divisor on $X$ of degree $d$ and $L$ the corresponding holomorphic line bundle $\mathscr{O}_{X}(D)$ over $X$ of degree $d$. Then the holomorphic line bundle $\sigma^{*} \bar{L}$ corresponds to the divisor $\sigma(D)$, that is, $\sigma^{*} \bar{L} \cong \mathscr{O}_{X}(\sigma(D))$.

Proof. Since $L \cong \mathscr{O}_{X}(D)$, we have a meromorphic section $s$ of $L$ with the positive part of $D$ as the zeros of $s$ (of order given by multiplicity) and the negative part of $D$ as the poles of $s$ (of order given by multiplicity). Since $L$ and $\bar{L}$ are identified as real rank two vector bundles, the pullback $\sigma^{*} s$ defines a smooth section of $\sigma^{*} \bar{L}$ over the complement (in $X$ ) of the support of $D$. 
It is straight-forward to check that the section $\sigma^{*} s$ of $\sigma^{*} \bar{L}$ is meromorphic. The divisor defined by the meromorphic section $\sigma^{*} s$ clearly coincides with $\sigma(D)$. Consequently, $\sigma^{*} \bar{L}$ is holomorphically isomorphic to the line bundle over $X$ defined by the divisor $\sigma(D)$. This completes the proof of the proposition.

Recall the quotient space $J_{0}(X):=\operatorname{Div}_{0}(X) / \operatorname{Div}_{P}(X)$ considered in 2 . The Jacobian $J_{0}(X)$ is identified with the space of all isomorphism classes of degree zero holomorphic line bundles over $X$. The isomorphism sends any divisor $D$ to the line bundle $\mathscr{O}_{X}(D)$. As in 3. let $\sigma_{0}$ denote the involution of $J_{0}(X)$ defined by $\sigma$. From Proposition 4.1 it follows immediately that the above identification of $J_{0}(X)$ with degree zero line bundles takes the involution $\sigma_{0}$ to the involution defined by $L \longmapsto \sigma^{*} \bar{L}$ on the space of all isomorphism classes of degree zero line bundles.

Let $D$ be a divisor of degree zero on the nonorientable Klein surface $Y$. From Proposition 2.5 it follows immediately that $D$ is principal if and only if $\pi^{*} D$ is principal. Therefore, we have an injective homomorphism

$$
\rho: \frac{\operatorname{Div}_{0}(Y)}{\operatorname{Div}_{P}(Y)} \longrightarrow \frac{\operatorname{Div}_{0}(X)}{\operatorname{Div}_{P}(X)}=J_{0}(X)
$$

defined by $D \longmapsto \pi^{*} D$, where $\operatorname{Div}_{P}(Y)$ denotes the group of principal divisors on $Y$ (as before, Div $_{0}$ denotes degree zero divisors).

Theorem 4.2. The image of the homomorphism $\rho$ in (3) coincides with the subgroup of $J_{0}(X)$ defined by all holomorphic line bundle $L$ with $\sigma^{*} \bar{L}$ holomorphically isomorphic to $L$.

Proof. Let $D$ be a divisor on $Y$ of degree zero. The divisor $\pi^{*} D$ on $X$ is left invariant by the action of the involution $\sigma$. From the above remark that the involution $\sigma_{0}$ is taken into the involution defined by $L \longmapsto \sigma^{*} \bar{L}$, it follows immediately that the holomorphic line bundle $L=\mathscr{O}_{X}\left(\pi^{*} D\right)$ over $X$ corresponding to the divisor $\pi^{*} D$ satisfies the condition $L \cong \sigma^{*} \bar{L}$.

For the converse direction, take a holomorphic line bundle $L$ over $X$ which has the property that $\sigma^{*} \bar{L}$ is isomorphic to $L$. Let $s$ be a nonzero meromorphic section of $L$. If the $\operatorname{divisor} \operatorname{div}(s)$ is left invariant by the involution $\sigma$, then $L$ is in the image of $\rho$.

If $\operatorname{div}(s)$ is not left invariant by the involution $\sigma$, then consider the meromorphic section of $\sigma^{*} \bar{L}$ defined by $\sigma^{*} s$. (Recall that $\sigma^{*} \bar{L}$ and $\sigma^{*} L$ are identified as real rank two $C^{\infty}$ bundles, and the section of $\sigma^{*} \bar{L}$ defined by $\sigma^{*} s$ using this identification is meromorphic.)

Now, fix a holomorphic isomorphism

$$
\alpha: L \longrightarrow \sigma^{*} \bar{L}
$$

such that the composition

$$
L \stackrel{\alpha}{\longrightarrow} \sigma^{*} \bar{L} \stackrel{\sigma^{*} \bar{\alpha}}{\longrightarrow} \overline{\sigma^{*} \sigma^{*} \bar{L}}=L
$$

is the identity automorphism of $L$, where $\bar{\alpha}$ is the isomorphism of $\bar{L}$ with $\overline{\sigma^{*} \bar{L}}$ induced by $\alpha$. Note that such an isomorphism exists. Indeed, if

$$
\alpha^{\prime}: L \longrightarrow \sigma^{*} \bar{L}
$$

is any isomorphism, then the automorphism $\sigma^{*} \overline{\alpha^{\prime}} \circ \alpha^{\prime}$ of $L$ (defined as in (5)) is the multiplication by a nonzero scalar $c \in \mathbb{C}$. Take any $c_{0} \in \mathbb{C}$ such that $c_{0}^{2}=c$. Now the isomorphism $\alpha=\alpha^{\prime} / c_{0}$ satisfies the condition that the composition in 5 is the identity automorphism of $L$. 
Let $s^{\prime}$ be the meromorphic section of $L$ defined by the above section $\sigma^{*} s$ using this isomorphism. Consider the meromorphic section $s^{\prime}+s$ of $L$. Since $\operatorname{div}(s)$ is not left invariant by $\sigma$, this meromorphic section $s^{\prime}+s$ is not identically zero. The $\operatorname{divisor} \operatorname{div}\left(s+s^{\prime}\right)$ is clearly left invariant by the involution $\sigma$. Hence $L \in J_{0}(X)$ is in the image of $\rho$. This completes the proof of the theorem.

\section{Nonorientable line bundle}

In this section we will define a line bundle on $Y$ intrinsically without using $X$.

Let $\left\{U_{i}\right\}_{i \in I}$ be a covering of $Y$ by open subsets and for each $U_{i}$,

$$
\phi_{i}: U_{i} \longrightarrow \mathbb{R}^{2}
$$

a $C^{\infty}$ coordinate chart. Consider the trivial (real) line bundle $U_{i} \times \mathbb{R}$ on each $U_{i}$. Using

$$
\frac{\operatorname{det}\left(\phi_{j} \circ \phi_{i}^{-1}\right)}{\left|\operatorname{det} \mathrm{d}\left(\phi_{j} \circ \phi_{i}^{-1}\right)\right|} \in \pm 1 \subset \operatorname{Aut}(\mathbb{R})
$$

as the transition function over $U_{i} \cap U_{j}$ for the pair $(i, j)$, we get a real line bundle over $Y$. This line bundle will be denoted by $\xi$. Since the transition functions are \pm 1 , the line bundle $\xi^{\otimes 2}$ has a natural isomorphism with the trivial line bundle $Y \times \mathbb{R}$. Let

$$
\lambda: \xi^{\otimes 2} \longrightarrow Y \times \mathbb{R}
$$

be the isomorphism.

We will give a construction of the line bundle $\xi$ without using coordinate charts. Consider the complement $\wedge^{2} T Y \backslash\left\{0_{Y}\right\}$ of the zero section of the real line bundle $\wedge^{2} T Y$, where $T Y$ is the real tangent bundle of $Y$. The multiplicative group

$$
\mathbb{R}^{+}:=\{c \in \mathbb{R} \mid c>0\}
$$

acts on $\Lambda^{2} T Y \backslash\{0\}$. The action of any $c \in \mathbb{R}^{*}$ sends any $v \in \Lambda^{2} T Y \backslash\{0\}$ to $c v$. Also, the multiplicative group \pm 1 acts on $\bigwedge^{2} T Y \backslash\{0\}$ by sending any $v$ to $\pm v$. Since these two actions commute, we have an action of the multiplicative group \pm 1 on

$$
Z:=\frac{\bigwedge^{2} T Y \backslash\left\{0_{Y}\right\}}{\mathbb{R}^{+}}
$$

Now, we have

$$
\xi=\frac{Z \times \mathbb{R}}{ \pm 1}
$$

where \pm 1 acts diagonally and it acts on $\mathbb{R}$ as multiplication by \pm 1 .

We will show that the Klein surface (nonorientable complex) structure on $Y$ gives an isomorphism of $T Y$ with $T Y \otimes \xi$, where $T Y$ as before is the (real) tangent bundle of $Y$. To construct the isomorphism, take a compatible coordinate chart

$$
\phi_{i}: U_{i} \longrightarrow \mathbb{C}
$$

compatible with the nonorientable complex structure. The orientation of the complex line $\mathbb{C}$ induces an orientation of $U_{i}$ using $\phi_{i}$. This gives a trivialization of $\xi$ over $U_{i}$ (this 
induced trivialization is also clear from the first construction of $\xi$ ). Using $\phi_{i}$ we have a complex structure on $U_{i}$ obtained from the complex structure of $\mathbb{C}$. Let

$$
\gamma_{i}:\left.T U_{i} \longrightarrow T U_{i} \otimes \xi\right|_{U_{i}}
$$

be the isomorphism defined by the almost complex structure of $U_{i}$ and the trivialization of $\left.\xi\right|_{U_{i}}$. If $\phi_{j}$ is another compatible coordinate chart then the function $\phi_{i} \circ \phi_{j}^{-1}$ is either holomorphic or anti-holomorphic. This immediately implies that the isomorphism

$$
\gamma_{j}:\left.T U_{j} \longrightarrow T U_{j} \otimes \xi\right|_{U_{j}}
$$

(obtained by repeating the construction of $\gamma_{i}$ for the new compatible coordinate chart) coincides with $\gamma_{i}$ over $U_{i} \cap U_{j}$. Consequently, the locally defined isomorphisms $\left\{\gamma_{i}\right\}$ patch together compatibly to give a global isomorphism

$$
\gamma: T Y \longrightarrow T Y \otimes \xi
$$

over $Y$.

A nonorientable complex line bundle over $Y$ is a $C^{\infty}$ real vector bundle of rank two over $Y$ together with a $C^{\infty}$ isomorphism of vector bundles

$$
\tau: E \longrightarrow E \otimes \xi
$$

satisfying the condition that the composition

$$
E \stackrel{\tau}{\longrightarrow} E \otimes \xi \stackrel{\tau \otimes \mathrm{Id}_{\xi}}{\longrightarrow}(E \otimes \xi) \otimes \xi=E \otimes \xi \stackrel{\mathrm{Id}_{E} \otimes \lambda}{\longrightarrow} E
$$

coincides with the automorphism of $E$ defined by multiplication with -1 , where $\lambda$ is defined in (6).

Therefore, if for a point $y \in Y$ we fix $w \in \xi_{y}$ with $\lambda(w \otimes w)=1$, then the automorphism of the fiber $E_{y}$ defined by

$$
v \longmapsto\left\langle\tau(v), w^{*}\right\rangle
$$

is an almost complex structure on $E_{y}$, where $\langle-,-\rangle$ denotes the contraction of $\xi_{y}$ with its dual line $\xi_{y}^{*}$ and $w^{*} \in \xi_{y}^{*}$ is the dual element of $w$, that is, $\left\langle w, w^{*}\right\rangle=1$.

Let $(E, \tau)$ be a nonorientable complex line bundle over $Y$ as above. It is easy to see that the $C^{\infty}$ vector bundle $E$ is not orientable. Indeed, the two orientations on a twodimensional real vector space $V$ defined by $J$ and $-J$, where $J$ is an almost complex structure on $V$, are opposite to each other. To explain this, note that an orientation of the tangent space $T_{y} Y$, where $y \in Y$, induces an orientation of the fiber $E_{y}$ and conversely. Indeed, giving an orientation of $T_{y} Y$ is equivalent to giving a vector in $w \in \xi_{y}$ with $\lambda$ ( $w \otimes$ $w)=1$. As it was shown above, such an element $w$ gives an almost complex structure on $E_{y}$. Hence $E_{y}$ gets an orientation. Conversely, if we have an orientation of the fiber $E_{y}$, then choose the element $w \in \xi_{y}$, with $\lambda(w \otimes w)=1$, that induces this orientation using $\tau$. Now, $w$ gives an orientation of $T_{y} Y$. Therefore, giving an orientation of $E_{y}$ is equivalent to giving an orientation of $T_{y} Y$. Since the tangent bundle $T Y$ is not orientable, we conclude that the vector bundle $E$ is not orientable.

The total space of the vector bundle $E$ will also be denoted by $E$. Let

$$
f: E \longrightarrow Y
$$


be the natural projection. Note that the relative tangent bundle for $f$ (that is, the kernel of the differential $\mathrm{d} f$ ) is identified with $f^{*} E$. So we have the following exact sequence of vector bundle

$$
0 \longrightarrow f^{*} E \longrightarrow T E \longrightarrow f^{*} T Y \longrightarrow 0
$$

over the manifold $E$.

The line bundle $f^{*} \xi$ will be denoted by $\hat{\xi}$. Let

$$
J: T E \longrightarrow T E \otimes \hat{\xi}
$$

be an isomorphism such that the composition

$$
T E \stackrel{J}{\longrightarrow} T E \otimes \hat{\xi} \stackrel{J \otimes \mathrm{Id}_{\hat{\xi}}}{\longrightarrow}(T E \otimes \hat{\xi}) \otimes \hat{\xi}=T E \otimes \hat{\xi} \stackrel{\operatorname{Id}_{E} \otimes f^{*} \lambda}{\longrightarrow} T E
$$

coincides with the automorphism of $E$ defined by multiplication with -1 . Assume that the isomorphism $J$ satisfies the following further conditions:

(1) The subbundle $f^{*} E$ in (10) is preserved by $J$ and $\left.J\right|_{f^{*} E}$ coincides with the isomorphism $f^{*} \tau$, where $\tau$ is defined in 8 .

(2) The action of $J$ on the quotient $f^{*} T Y$ in (10) coincides with the isomorphism $f^{*} \gamma$, where $\gamma$ is constructed in (7).

A holomorphic structure on the nonorientable complex line bundle $E$ is an isomorphism $J$ as above satisfying the following conditions (apart from the above conditions) described below.

If we take a coordinate chart $(U, \phi)$ on $Y$ compatible with the nonorientable Riemann surface structure, then as we saw before, the restriction $\left.\xi\right|_{U}$ gets a trivialization. This in turn gives a trivialization of $\hat{\xi}$ over $f^{-1}(U)$. Using this trivialization of $\left.\hat{\xi}\right|_{f^{-1}(U)}$, the isomorphism $\left.J\right|_{f^{-1}(U)}$ becomes an automorphism $J_{\phi}$ of $(T E)_{f^{-1}(U)}$ with the property that $J_{\phi} \circ J_{\phi}$ coincides with the automorphism of $(T E)_{f^{-1}(U)}$ given by multiplication with -1 . In other words, $J_{\phi}$ is an almost complex structure on $f^{-1}(U)$.

A holomorphic structure on the nonorientable complex line bundle $E$ is an isomorphism $J$ satisfying the following two conditions (apart from the earlier conditions):

(1) The almost complex structure $J_{\phi}$ on $f^{-1}(U)$ is integrable for every compatible coordinate chart.

(2) There is a homomorphic isomorphism

$$
f_{\phi}: f^{-1}(U) \longrightarrow \phi(U) \times \mathbb{C} \subset \mathbb{C} \times \mathbb{C}
$$

that fits in a commutative diagram

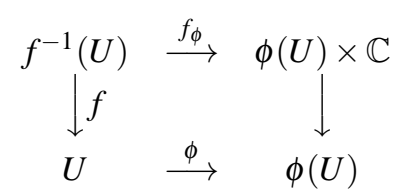

(the right vertical arrow is the projection to the first coordinate), and the restriction of $f_{\phi}$ to any fiber of $f$ is a complex linear isomorphism with $\mathbb{C}$. 
A holomorphic line bundle over $Y$ is defined to be a complex line bundle equipped with a holomorphic structure.

As in $\$ 2$ let $\pi: X \longrightarrow Y$ be the double cover of the nonorientable Riemann surface $Y$ given by local orientations. As before, let $\sigma$ denote the anti-holomorphic involution of the Riemann surface $X$.

Theorem 5.1. The space of all holomorphic line bundles over $Y$ are in bijective correspondence with the holomorphic line bundles $L$ over $X$ with the property that $\sigma^{*} \bar{L}$ is holomorphically isomorphic to $L$.

Proof. Let $L$ be a holomorphic line bundle over $X$ such that $\sigma^{*} \bar{L}$ is holomorphically isomorphic to $L$. Fix an isomorphism

$$
\alpha: L \longrightarrow \sigma^{*} \bar{L}
$$

as in (4) such that the composition in (5) is the identity automorphism of $L$.

Since the underlying $C^{\infty}$ line bundle for $\bar{L}$ is identified with that of $L$, the isomorphism $\alpha$ gives a $C^{\infty}$ isomorphism of $L$ with $\sigma^{*} L$ whose composition with itself is the identity automorphism of $L$. In other words, $\alpha$ is a $C^{\infty}$ lift to $L$ of the involution $\sigma$ of $X$. Therefore, the quotient $L / \alpha$ is a real vector bundle of rank two over $X / \sigma=Y$. This real vector bundle of rank two over $Y$ will be denoted by $E$.

To construct a complex structure on $E$, first note that the (real) line bundle $\pi^{*} \xi$ over $X$ is canonically trivialized, i.e., there is a natural isomorphism of $\pi^{*} \xi$ with the trivial line bundle $X \times \mathbb{R}$ over $X$. Indeed, this follows immediately from the definitions of $X$ and $\xi$. The complex structure on the fibers on $L$ give an isomorphism

$$
L \longrightarrow L
$$

defined by multiplication by $\sqrt{-1}$. Consider the composition

$$
L \longrightarrow L \longrightarrow L \otimes_{\mathbb{R}}(X \times \mathbb{R}) \longrightarrow L \otimes_{\mathbb{R}} \pi^{*} \xi
$$

which we denote by $J_{0}$. Since $\pi^{*} \xi$ is the pullback of a line bundle over $Y$, there is a natural lift of the involution $\sigma$ to $\pi^{*} \xi$. On the other hand, $\alpha$ is a $C^{\infty}$ lift of the involution $\sigma$ to $L$. Therefore, we have a lift of the involution $\sigma$ to $L \otimes_{\mathbb{R}} \pi^{*} \xi$. It is straight-forward to check that the isomorphism $J_{0}$ defined above commutes with the lifts of the involution $\sigma$ to $L$ and $L \otimes_{\mathbb{R}} \pi^{*} \xi$. This immediately implies that the isomorphism $J_{0}$ descends to an isomorphism of $E$ with $E \otimes_{\mathbb{R}} \xi$ over $Y$. This isomorphism of $E$ with $E \otimes_{\mathbb{R}} \xi$, which we denote by $J$, clearly satisfies the condition that the composition in (9) is multiplication by -1 . Therefore, $(E, J)$ is a nonorientable complex line bundle.

It is easy to see that $J$ defines a holomorphic structure on $E$. Indeed, this is an immediate consequence of the fact that the almost complex structure on the total space of $L$ is integrable.

For the converse direction, take a holomorphic line bundle $(E, J)$ over $Y$. Consider the (real) rank two $C^{\infty}$ vector bundle $\pi^{*} E$ over $X$. Since $\pi^{*} \xi$ is identified with the trivial line bundle, the complex structure $\tau$ on $E$ (defined in $(8)$ ) gives a complex structure on $\pi^{*} E$. For the same reason, $J$ defines an integrable complex structure on the total space of $\pi^{*} E$. Using the conditions on $J$ the vector bundle $\pi^{*} E$ gets the structure of a holomorphic line bundle over $X$. 
Since $\pi^{*} E$ is the pullback of a vector bundle over $Y$, the involution $\sigma$ of $X$ has a natural $C^{\infty}$ lift to $\pi^{*} E$. The isomorphism of $\pi^{*} E$ with $\sigma^{*} \pi^{*} E$ defined by this lift gives a holomorphic isomorphism of the holomorphic line bundle $\pi^{*} E$ with $\sigma^{*} \overline{\pi^{*} E}$. This completes the proof of the theorem.

\section{References}

[1] Arés Gastesi P, A note on Torelli spaces of non-orientable compact Klein surfaces, Ann. Acad. Sci. Fenn. Math. 24 (1999) 23-30

[2] Farkas H and Kra I, Riemann Surfaces, Volume 72 of Graduate Text in Mathematics, 2nd edition (New York, Heidelberg and Berlin: Springer-Verlag) (1992) 